\title{
A 4-CHROMATIC GRAPH WITH A SPECIAL PLANE DRAWING
}

\author{
NICHOLAS WORMALD
}

(Received 15 May; revised 25 October 1978)

Communicated by W. D. Wallis

\begin{abstract}
A graph is found which is 4-chromatic, has girth 5 , and can be obtained by taking a set $S$ of points in the plane and joining two of the points whenever their distance is 1 . The points in $S$ are not found explicitly, but their existence is demonstrated by use of a computer.
\end{abstract}

Subject classification (Amer. Math. Soc. (MOS) 1970): 05 C 15, 05 C 10.

Suppose two points in the plane are joined whenever their distance is 1 . A wellknown problem of Nelson asks for the chromatic number of the resultant graph. The number was shown to be at least 4 by $L$. and W. Moser (1961), who exhibited the example shown in Fig. 1, whilst the upper bound 7 follows from Hadwiger $e$ al. (1964).

Erdös has modified the problem as follows. Let $S$ be a subset of the plane which contains no equilateral triangle with side 1 . Join two points of $S$ if and only if their distance is 1 . Does this graph always have chromatic number at most three? If the answer is no, assume that the graph defined by $S$ contains no $C_{r}$ for $3 \leqslant r \leqslant t$ and ask the same question.

By demonstrating the existence of a set $S$ which defines a 4-chromatic graph $G$ with girth 5 , we answer this question in the negative for $t=3$ and 4. Basic graphtheoretic notation can be found in Harary (1969).

The graph $G$ is too large to be drawn here, but can be constructed as follows. Let $H$ be a 5 -cycle with its points labelled 1 through 5 in cyclic order, and let $R$ be a set of 13 points with an associated linear order. For each 5-subset $U$ of $R$, the ordering of the points in $R$ induces a linear order on the points of $U$, and we attach

(C) Copyright Australian Mathematical Society 1979

Copyright. Apart from any fair dealing for scholarly purposes as permitted under the Copyright Act, no part of this JOURNAL may be reproduced by any process without written permission from the Treasurer of the Australian Mathematical Society. 
a copy $H_{U}$ of $H$ to $U$ by joining each of the points 1 through 5 in $H_{U}$ to the corresponding point in the linear order of $U$. The resultant graph $G$ has $13+5\left(\begin{array}{c}13 \\ 5\end{array}\right)=6448$ points, 13 of which have degree 495 , with the remainder all being of degree 3 .

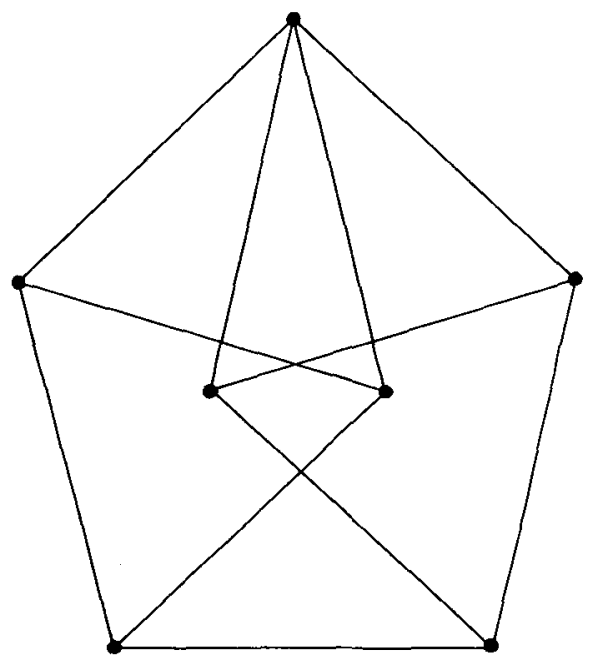

FIGURE 1. A 4-chromatic graph.

$G$ is 4-colourable, since the points of the copies of $H$ in $G$ can be coloured with three colours and the points in $R$ with the fourth colour. In a hypothetical 3-colouring of $G$, however, some 5-subset $U$ of $R$ must be monochromatic, and consequently the points of the 5-cycle $H_{U}$ must be coloured in two colours only. This contradiction implies that $G$ is 4-chromatic. Moreover, it is clear that $G$ contains neither a triangle nor a 4-cycle.

It must be shown that for some subset $S$ of the plane, the graph $G$ is formed by joining two points of $S$ whenever the distance between them is 1 . The sequence $u_{0}, \ldots, u_{4}$ of points is pentagonizable if there is some set $\left\{v_{0}, \ldots, v_{4}\right\}$ of points which are the vertices of a pentagon with unit sides, such that $u_{i}$ and $v_{i}$ have distance 1 for each $i$. The points $v_{0}, \ldots, v_{4}$ then pentagonize $u_{0}, \ldots, u_{4}$. Our main obstacle is the problem of finding suitably general conditions on a sequence $u_{0}, \ldots, u_{4}$ which are sufficient for it to be pentagonizable. In our solution to this problem we do not find the set $\left\{v_{0}, \ldots, v_{4}\right\}$ explicitly. Indeed, to do this in general appears to be very difficult, because the constraints on the positions of the points call for various distances to be precisely 1 . We overcome this difficulty by using continuity arguments to show that a suitable set $\left\{v_{0}, \ldots, v_{4}\right\}$ exists whenever $u_{0}, \ldots, u_{4}$ satisfy certain conditions. These new conditions require various distances between points to satisfy inequalities but not equalities. This allows us to use a computer for 
solving the problem: all we need to do is ensure that the rounding errors, generated by representing real numbers using a finite number of digits, are so small that they have no effect on the truth of any of the inequalities which we desire to hold true. For example, if we require a number $x$ to be less than 1 , and it is known that the relative error in the calculated value, $y$ say, of $x$ is less than $10^{-7}$, it is enough to demand that $y<1-10^{-6}$, say.

The continuity arguments which we shall use require a substantial introduction. Throughout this paper each angle is identified with its radian measure $r$ which is taken in the range $0 \leqslant r<2 \pi$. If $u, v$ and $w$ are points in the plane, the directed line passing from $u$ to $v$ is denoted by $u v$, and the angle $u v w$ is the angle from $v u$ to $v w$. A point $v^{\prime}$ is acceptable to the ordered triple $\left(u, u^{\prime}, v\right)$ of points if

(i) both the angle $\alpha$ from $u v$ to $u^{\prime} v^{\prime}$ and the angle $\beta=u^{\prime} v^{\prime} v$ are strictly between 0 and $\pi$, and

(ii) $v^{\prime}$ has distance 1 from $u^{\prime}$.

The concept of acceptability of a point is illustrated in Fig. 2. If $u_{0}, \ldots, u_{n}$ and $v_{0}, \ldots, v_{n}$ are two sequences of points in the plane and $v_{i}$ is acceptable to the triple $\left(u_{i-1}, u_{i}, v_{i-1}\right)$ for $1 \leqslant i \leqslant n$ then the sequence $v_{0}, \ldots, v_{n}$ is acceptable to the sequence $u_{0}, \ldots, u_{n}$.

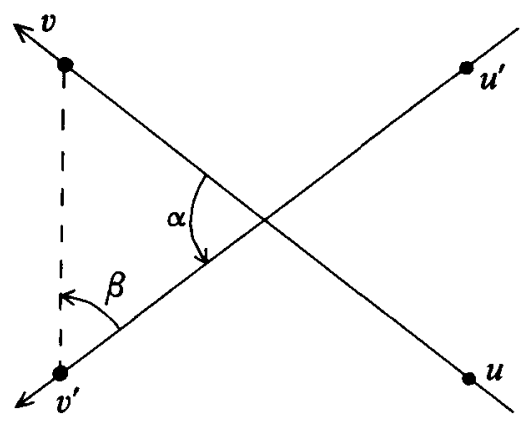

FIGURE 2. A configuration of points in which $v^{\prime}$ is acceptable to $\left(u, u^{\prime}, v\right)$.

For any two distinct points $u^{\prime}$ and $v$ in the plane with distance less than 2, there is a unique point $v^{\prime}$ with distance 1 from both $u^{\prime}$ and $v$ such that the angle $u^{\prime} v^{\prime} v$ is strictly between 0 and $\pi$. The point $v^{\prime}$ is called the trailer of $v$ from $u^{\prime}$.

We state without proof the following elementary geometrical result, which will be used when we come to show that a certain sequence of points is pentagonizable.

LEMMA. Let $u$ and $u^{\prime}$ be two points in the plane with distance less than 1 , and let $v$ and $w$ be two points both with distance 1 from $u$. If $v^{\prime}$ and $w^{\prime}$ are the trailers of $v$ and $w$ respectively from $u^{\prime}$, then the angle vuw plus the angle from $u w$ to $u^{\prime} w^{\prime}$ is greater than the angle from $u v$ to $u^{\prime} v^{\prime}$. 
Again suppose that $u_{0}, \ldots, u_{n}$ and $v_{0}, \ldots, v_{n}$ are two sequences of points in the plane. For $1 \leqslant i \leqslant n$ let $\alpha_{i}$ be the angle from $u_{i-1} v_{i-1}$ to $u_{i} v_{i}$. The wrap of the sequence $v_{0}, \ldots, v_{n}$ around the sequence $u_{0}, \ldots, u_{n}$ is the sum of the angles $\alpha_{i}$, for $1 \leqslant i \leqslant n$. Note that if $u_{0}=u_{n}$ and $v_{0}=v_{n}$, this wrap is necessarily a multiple of $2 \pi$.

Now suppose $j<n$, and let $u_{0}, \ldots, u_{n}$ and $v_{0}, \ldots, v_{j}$ be two sequences of points in the plane such that the distance between $u_{i-1}$ and $u_{i}$ is less than 1 for $1 \leqslant i \leqslant n$, and the distance between $v_{j}$ and $u_{j+1}$ is less than 2 . For $j<i \leqslant n$, let $v_{i}$ be the trailer of $v_{i-1}$ from $u_{i}$. The resultant sequence $v_{0}, \ldots, v_{n}$ is the trailing completion of $v_{0}, \ldots, v_{j}$ from $u_{0}, \ldots, u_{n}$.

Let $T$ be a set of 13 distinct points in the plane with the distance between any two points in $T$ less than 1 , and let $\varepsilon$ be a positive number. Choose a relation, denoted by $<$, on $T$ which orders $T$ totally. Suppose that for each 5-subset $U=\left\{u_{0}, \ldots, u_{4}\right\}$ of $T$ with $u_{0}<\ldots<u_{4}$ there are two sequences $V^{\prime}=v_{0}^{\prime}, \ldots, v_{4}^{\prime}$ and $V^{\prime \prime}=v_{0}^{\prime \prime}, \ldots, v_{4}^{\prime \prime}$ of points satisfying the following conditions.

(i) The sequences $v_{0}^{\prime}, \ldots, v_{4}^{\prime}, v_{0}^{\prime}$ and $v_{0}^{\prime \prime}, \ldots, v_{4}^{\prime}, v_{0}^{\prime \prime}$ are acceptable to the sequence $u_{0}, \ldots, u_{4}, u_{0}$.

(ii) For $0 \leqslant i \leqslant 4$, the points $v_{i}^{\prime}$ and $v_{i+1}^{\prime}$ have distance at most $1-\varepsilon$, and the points $v_{i}^{\prime \prime}$ and $v_{i+1}^{\prime \prime}$ have distance at least $1+\varepsilon$, subscripts being taken modulo 5 .

(iii) The wrap of both $v_{0}^{\prime}, \ldots, v_{4}^{\prime}, v_{0}^{\prime}$ and $v_{0}^{\prime \prime}, \ldots, v_{4}^{\prime \prime}, v_{0}^{\prime \prime}$ around $u_{0}, \ldots, u_{4}, u_{0}$ is $2 \pi$. These properties of $T$ will be referred to as $(C)$. We shall show that $(C)$ implies the existence of the required subset $S$ of the plane. Consideration of the existence question for the sequences $V^{\prime}$ and $V^{\prime \prime}$ is postponed until last.

Our first object is to show that for each 5 -subset $U=\left\{u_{0}, \ldots, u_{4}\right\}$ of $T$ with $u_{0}<\ldots<u_{4}$, the sequence $u_{0}, \ldots, u_{4}$ is pentagonizable. We define a function $f$ whose domain is the set of points on the unit circle centred at $u_{0}$. For any point $w_{0}$ in $\operatorname{dom} f$, let $w_{0}, \ldots, w_{5}$ be the trailing completion of $w_{0}$ from $u_{0}, \ldots, u_{4}, u_{0}$ and define $f\left(w_{0}\right)$ to be the wrap of $w_{0}, \ldots, w_{5}$ around $u_{0}, \ldots, u_{4}, u_{0}$. It is clear that $f$ is continuous. This point is vital to our argument, for we shall show that $f\left(v_{0}^{\prime}\right)>2 \pi$ and $f\left(v_{0}^{\prime \prime}\right)<2 \pi$, implying that there is some point $v_{0}$ such that $f\left(v_{0}\right)=2 \pi$. Thus, if $v_{0}, \ldots, v_{5}$ is the trailing completion of $v_{0}$ from $u_{0}, \ldots, u_{4}, u_{0}$, it follows that $v_{5}=v_{0}$. Hence $\left\{v_{0}, \ldots, v_{4}\right\}$ are the vertices of a pentagon with unit sides and consequently $u_{0}, \ldots, u_{4}$ is pentagonizable.

It is convenient to define $u_{5}$ to be the point $u_{0}$ and $v_{5}^{\prime}$ to be the point $v_{0}^{\prime}$. For $0 \leqslant i \leqslant 4$, let $v(i, 0), \ldots, v(i, 5)$ be the trailing completion of $v_{0}^{\prime}, \ldots, v_{i}^{\prime}$ from $u_{0}, \ldots, u_{5}$ and let $r_{i}$ be the wrap of $v(i, 0), \ldots, v(i, 5)$ around $u_{0}, \ldots, u_{5}$. Similarly let $v(5,0), \ldots, v(5,5)$ be the sequence $v_{0}^{\prime}, \ldots, v_{5}^{\prime}$, and let $r_{5}$ be the wrap of $v(5,0), \ldots, v(5,5)$ around $u_{0}, \ldots, u_{5}$. By definition, $r_{0}=f\left(v_{0}^{\prime}\right)$ and $r_{5}=2 \pi$. We shall show that $f\left(v_{0}^{\prime}\right)>2 \pi$ by proving that $r_{i-1}>r_{i}$ for $1 \leqslant i \leqslant 5$.

For $i \leqslant j \leqslant 5$ let $r(i, j)$ be the wrap of $v(i, 0), \ldots, v(i, j)$ around $u_{0}, \ldots, u_{j}$. To show $r(i-1,5)>r(i, 5)$, it is enough to show $r(i-1, j)>r(i, j)$ for all $i$ and $j$. This is done by induction on $j$ with $i$ fixed. First, suppose $j=i$. Then $v(i-1, j)=v(i-1, i)$ is 
the trailer of $v_{i-1}^{\prime}$ from $u_{i}$, and the sequences

$$
v(i, 0), \ldots, v(i, i) \text { and } v(i-1,0), \ldots, v(i-1, i)
$$

are just the sequences $v_{0}^{\prime}, \ldots, v_{i}^{\prime}$ and $v_{0}^{\prime}, \ldots, v_{i-1}^{\prime}, v(i-1, i)$ respectively. Since $v_{0}^{\prime}, \ldots, v_{5}^{\prime}$ is acceptable to $u_{0}, \ldots, u_{5}$, the point $v_{i}^{\prime}$ is acceptable to the ordered triple $\left(u_{i-1}, u_{i}, v_{i-1}^{\prime}\right)$. This, together with the assumptions that the points $v_{i-1}^{\prime}$ and $v_{i}^{\prime}$ have distance at most $1-\varepsilon$, that the points $u_{i-1}$ and $u_{i}$ have distance less than 1 , and that $v(i-1, i)$ is the trailer of $v_{i-1}^{\prime}$ from $u_{i}$, is enough to ensure that the angle from $u_{i-1} v_{i-1}^{\prime}$ to $u_{i} v_{i}^{\prime}$ is less than the angle from $u_{i-1} v_{i-1}^{\prime}$ to $u_{i} v(i-1, i)$. Hence, the wrap of $v_{0}^{\prime}, \ldots, v_{i-1}^{\prime}, v(i-1, i)$ around $u_{0}, \ldots, u_{i}$ is greater than the wrap of $v_{0}^{\prime}, \ldots, v_{i}^{\prime}$ around $u_{0}, \ldots, u_{i}$. Thus $r(i-1, i)>r(i, i)$ as required.

Now suppose $i<j \leqslant 5$ and assume $r(i-1, j-1)>r(i, j-1)$. Note that $r(i-1, j-1)$ is the angle from $u_{0} v_{0}^{\prime}$ to $u_{j-1} v(i-1, j-1)$ plus some non-negative multiple of $2 \pi$. Similarly, $r(i, j-1)$ is the angle from $u_{0} v_{0}^{\prime}$ to $u_{j-1} v(i, j-1)$ plus some non-negative multiple of $2 \pi$. It follows that $r(i-1, j-1)$ is $r(i, j-1)$ plus some non-negative multiple of $2 \pi$ plus the angle $v(i, j-1) u_{j-1} v(i-1, j-1)$. Denoting the latter angle by $\alpha$, we conclude that $r(i-1, j-1) \geqslant r(i, j-1)+\alpha$. As $j>i$, the points $v(i-1, j)$ and $v(i, j)$ are the trailers of $v(i-1, j-1)$ and $v(i, j-1)$ respectively from $u_{j}$. Let $\beta$ denote the angle from $u_{j-1} v(i-1, j-1)$ to $u_{j} v(i-1, j)$ and let $\gamma$ denote the angle from $u_{j-1} v(i, j-1)$ to $u_{j} v(i, j)$. The Lemma now implies that $\alpha+\beta>\gamma$. But $r(i-1, j)=r(i-1, j-1)+\beta$ by definition, and thus

$$
r(i-1, j) \geqslant r(i, j-1)+\alpha+\beta>r(i, j-1)+\gamma=r(i, j) .
$$

This establishes the inductive step, and we therefore have

$$
r_{i-1}=r(i-1,5)>r(i, 5)=r_{i}
$$

as required.

We have just finished showing that $f\left(v_{0}^{\prime}\right)>2 \pi$. The corresponding result that $f\left(v_{0}^{\prime \prime}\right)<2 \pi$ is readily established by similar arguments. This completes the proof that the sequence $u_{0}, \ldots, u_{4}$ is pentagonizable. Recall that $v_{0}, \ldots, v_{4}$ are the vertices of a corresponding pentagon; let $V=\left\{v_{0}, \ldots, v_{4}\right\}$. For each 5 -subset $U_{i}$ of $T$, a similar set $V_{i}$ can be found. Now let $W$ be the union of the $V_{i}$ over all 5 -subsets $U_{i}$ of $T$. As any two points in $T$ have distance less than 1 , none of the sets $V_{i}$ can contain a point in $T$. Therefore, if $V_{i}$ and $V_{j}$ are disjoint sets whenever $U_{i} \neq U_{j}$ it follows that the graph $G$ can be formed by joining selected pairs of points in $W \cup T$ which have distance 1 .

The problem is, there is no guarantee as yet that $V_{i}$ and $V_{j}$ are in fact disjoint for $U_{i} \neq U_{j}$ and even then it is possible that there are pairs of points in $W \cup T$ with distance 1 which correspond to non-adjacent points of $G$. For instance, a point in $V_{i}$ and a point in $V_{j}$ may have distance 1 . We deal with both these problems next. Generally speaking, the solution is to move the points of $T$ selectively through 
small distances so that points in just one of the sets $V_{i}$ and $V_{j}$ are moved. All other sets $V_{k}$ are adjusted accordingly.

First we have a few definitions. The function $f\left(w_{0}\right)$ clearly has a derivative $f^{\prime}\left(w_{0}\right)$ for all $w_{0}$ in $\operatorname{dom} f$. If $f^{\prime}\left(v_{0}\right)=0$, we say $V$ is singular. Suppose that $v_{0}, \ldots, v_{4}$ are all distinct, that the point $v_{i}$ has distance 1 from the point $t$ in $T$ if and only if $t=u_{i}$, and moreover that $v_{i}$ and $v_{j}$ have distance 1 if and only if $|i-j|=1$ or 4 . We then say $V$ is self-consistent. Also, if $U_{i}$ and $U_{j}$ are two 5-subsets of $T$, then $V_{i}$ is consistent with $V_{j}$ if no point of $V_{i}$ is a point of $V_{j}$ or has distance 1 from a point of $V_{j}$.

Define a total order < on the 5-subsets of $T$. By moving the points of $T$ when necessary, we plan to progress through the 5-subsets of $T$ in ascending order, making each 5-subset first non-singular and self-consistent and then consistent with all lesser 5-subsets of $T$. We need to have an idea of how the sets $V_{i}$ are affected when a point of $T$ is moved slightly. Suppose $V$ is not singular. Then if for some $i, u_{i}$ is moved a distance $\delta$ in any direction, it is possible to move the points $v_{0}, \ldots, v_{4}$ so that they still pentagonize the sequence $u_{0}, \ldots, u_{4}$, as long as $\delta$ is small enough. Moreover, because $V$ is not singular it can be arranged that the distances through which the points $v_{0}, \ldots, v_{4}$ move are as small as you like, by decreasing $\delta$ sufficiently. Both these results follow from the fact that $f$ is continuous when regarded as a function of the points $w_{0}, u_{0}, \ldots, u_{4}$, on the domain under consideration. Furthermore, in this context the partial derivative of $f$ with respect to $w_{0}$ is also continuous and so the new set $V$ is non-singular when $\delta$ is small enough.

There are two special types of operation which we shall employ to move a point of $T$. Operation 1 , as applied to a point $u_{i}$ in $U$, consists of moving the point $u_{i}$ through some distance $\delta$ in a direction towards $v_{i}$. If $\delta$ is sufficiently small, each of the points $v_{0}, \ldots, v_{4}$ must also be moved because each is the trailer of the one before it in the 5-cycle. Operation 2 is applied to $u_{i}$ by moving it through a distance $\delta$ so that it still has distance 1 from $v_{i}$. Consequent $\hat{y}$, no points of $V$ are moved in this operation. To avoid complicated notation, after any move of a point of $T$ we refer to the new set as $T$ and preserve the names of all corresponding points and sets.

We now begin our progression through the 5-subsets of $T$. The subscripts of the $v$ 's are taken modulo 5. First, let $U_{1}$ be the least 5-subset of $T$, and suppose $V_{1}$ is singular. For some $i$, we must have that $v_{i-1}$ is not $v_{i+1}$ and that the points $v_{i-1}$, $v_{i}$ and $v_{i+1}$ are not collinear. Application of operation 2 to $u_{i}$, with $\delta$ small, will now force $V_{1}$ to be non-singular. We next suppose that $V_{1}$ is not self-consistent. If $v_{0}, \ldots, v_{4}$ are not all distinct, we may assume $v_{i}=v_{i+2}$, and application of operation 1 to the point $u_{i+1}$ is enough to change this condition. If $v_{i}$ and $v_{j}$ have distance 1 where $|i-j|$ is not 1 or 4 , then application of operation 1 to any point $u_{k}$ in $U$ other than $u_{i}$ and $u_{j}$ moves both $v_{i}$ and $v_{j}$. A slight problem arises here if $v_{i}$ and $v_{j}$ remain unit distance apart no matter how small $\delta$ is made in this application of operation 1. This problem is overcome by applying operation 2 to $u_{i}$ or $u_{j}$ before the application of operation 1 to $u_{k}$. Next suppose $v_{i}$ and $t$ have distance 1 
when $t \neq u_{i}$ is in $T$. If $t$ is in $U$, apply operation 2 to $t$; otherwise move $t$ through an arbitrarily small distance in a direction away from $v_{i}$. In this way, $V$ can be made self-consistent.

The set $V_{2}$ corresponding to the least 5 -subset $U_{2}$ of $T$ greater than $U_{1}$ can now be made non-singular and self-consistent in the same way. Then, if a point in $V_{2}$ is in $V_{1}$ or has distance 1 from a point in $V_{1}$, application of operation 1 to any point in $U_{2}$ which is not in $U_{1}$ will remedy the situation. In this way $V_{2}$ can be made consistent with $V_{1}$. The process is repeated for each 5-subset of $T$ in turn, so that eventually each is self-consistent and consistent with all others. The set $W \cup T$ will then suffice for $S$.

There are two things we must watch out for in the process of moving points of $T$. Firstly, we must ensure that at no stage do we spoil the work we have already done. We have mentioned that if the movement of $u_{i}$ is made small enough, a non-singular $V$ will remain non-singular, and the movement in the points $v_{i}$ can be reduced as much as is desired. Note that the points in $T$ and in the sets $V_{i}$, together with all points of unit distance from any of these, form a closed subset of the plane. It follows that there is some positive number $\delta^{\prime}$ such that any movement of a point of $T$ through a distance less than $\delta^{\prime}$ can be effected without destroying consistency or self-consistency of the sets $V_{i}$ for $i \leqslant j$. If there are a number of places of inconsistency between $V_{i}$ and $V_{j}$, or of $V_{j}$ with itself, they can be treated one by one for the same reasons.

The second difficulty in the above process arises when we have progressed to the 5-subset $U$ of $T$ and one of the points of $U$ has already been moved. How do we know that $U$ is still pentagonizable? It is easily shown that there is some positive $\delta^{\prime \prime}$ such that when one of the points of $T$ is moved in any direction through a distance less than $\delta^{\prime \prime}$, the property $(C)$ of $T$ is retained. Therefore, this second difficulty is resolved by taking $\delta<\delta^{\prime \prime}$ in operations 1 and 2 , for this ensures that all 5-subsets of $T$ will remain pentagonizable. Of course, after each movement of a point of $T$, the sizes of $\delta^{\prime}$ and $\delta^{\prime \prime}$ may be modified.

All that remains is to find a set $T$ satisfying the property $(C)$. Let $T$ be the set of vertices of a regular 13-gon on a circle with centre $z$ and radius 0.49 . The maximum distance between two points of $T$ is certainly less than 1 . Denote the points in $T$ by $t_{0}, \ldots, t_{12}$ so that the angle $t_{i} z t_{i+1}$ is $2 \pi / 13$ for each $i$, and then put $t_{0}<\ldots<t_{12}$. A computer search was undertaken to find sets $V^{\prime}$ and $V^{\prime \prime}$ for each subset $U=\left\{u_{0}, \ldots, u_{4}\right\}$ of $T$, with $\varepsilon$ taken as $10^{-3}$. Candidates which were considered for $v_{j}^{\prime}$ and $v_{j}^{\prime \prime}$ were those points $w$ with distance 1 from $u_{j}$ such that the angle from $z t_{0}$ to $u_{j} w$ is a multiple of $2 \pi / n$, for various $n$. The programming was designed so that the rounding errors generated by representing real numbers using a finite number of digits could have no effect in the final result. Of course, only one set $U$ from each class of congruent subsets of $T$ needed to be considered, as the existence of $V^{\prime}$ and $V^{\prime \prime}$ depends only on the relative positions of the points in $U$. Suitable 
sets $V^{\prime}$ were found with $n=18$ for all $U$ considered, whilst $n=9$ was sufficient in the case of $V^{\prime \prime}$. Only the solutions for $U=\left\{t_{0}, \ldots, t_{4}\right\}$ will be given here. In this case the angles from $z t_{0}$ to $u_{j} v_{j}^{\prime}$, for $j=0, \ldots, 4$, are the following multiples of $\pi / 9$ : $5,8,11,14,17$. This defines $V^{\prime}$. The corresponding multiples of $2 \pi / 9$ which define $V^{\prime \prime}$ are 6, 8, 1, 3 and 5. These sets $V^{\prime}$ and $V^{\prime \prime}$ are illustrated in Fig. 3. A full list of solutions can be obtained from the author.

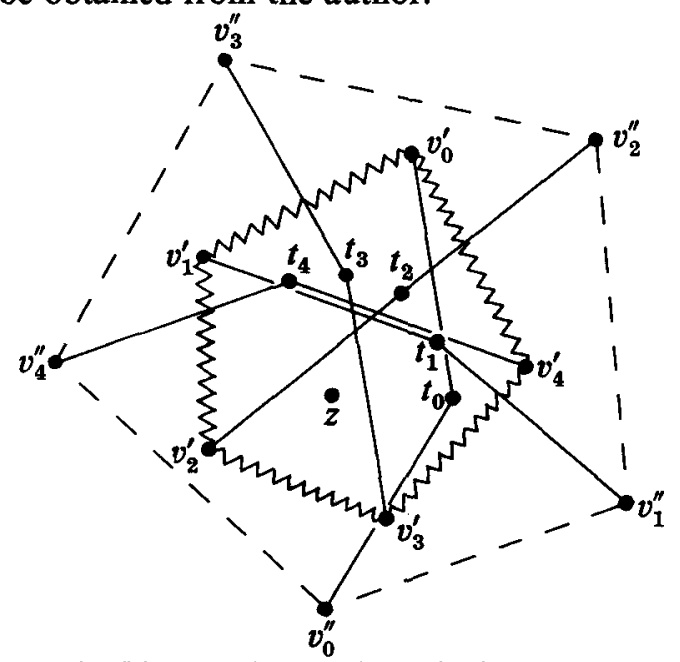

Figure 3. The sets $V^{\prime}$ and $V^{\prime \prime}$ for $U=\left\{t_{0}, \ldots, t_{4}\right\}$. Unit distances are represented by solid lines. Distances which are required to be less or greater than 1 are represented by zig-zag lines and broken lines respectively.

One can now ask (as Erdös has) whether there is a set $S$ in the plane which defines a 4-chromatic graph $G$ with no 3-, 4- or 5-cycles. It is conceivable that our methods could be adapted to prove the existence of such a set, by letting $T$ have 19 points and using 7-cycles attached to the 7-subsets of $T$, instead of 5-cycles attached to 5-subsets. We do not pursue the matter further, however, for the progression to a graph $G$ with girth greater than 6 appears to require a different form of attack.

\section{References}

H. Hadwiger, H. Debrunner and V. Klee (1964), Combinatorial geometry in the plane (Holt, Rinehart \& Winston, New York).

F. Harary (1969), Graph theory (Addison-Wesley, Reading, Mass.).

L. Moser and W. Moser (1961), 'Solution to Problem 10', Canad. Math. Bull. 4, 187-189.

Department of Mathematics

University of Newcastle

Newcastle, N.S.W. 2308

Australia 